\title{
Reasons Why a Paper Is Not Selected for Publication in the Acta Chirurgica Belgica
}

\author{
---by H. Van Damme, W. Ceelen (The Editors) - 1/06/2008
}

\section{Acta Chir Belg, 2008, 108, 281}

Manuscripts submitted for peer review publication may be rejected for a number of different reasons, most of which are avoidable.

It should be noted that the reasons for accepting manuscripts are not the mirror image of the reasons for rejecting manuscripts. The main reasons for accepting manuscripts are : their contribution and relevance to the field, excellence of writing, and quality of the study design.

Many journals expect reviewers to assess the scientific merits and validity of research in submitted manuscripts; however, reviewers can become critical of manuscripts containing numerous language errors, which are difficult to eliminate without careful editing. Scientific writing demands both good science and well written manuscripts.

Following are the principal reasons why manuscripts are rejected. They are all equally important because reviewers tend to focus on different issues depending on their individual concerns and the journal's requirements.

\begin{abstract}
1. Poor experimental design and/or inadequate investigation. An inadequate sample size, a biased sample, a non-unique concept, and scientific flaws in the study are common faults.
\end{abstract}

2. Failure to conform to the targeted journal. This is a common mistake. The focus of the manuscript is not within the scope of the journal and/or the guidelines of the targeted journal are not followed. This can easily be avoided by reading the targeted journal and reviewing the author guidelines.

3. Poor English grammar, style, and syntax. Though poor writing may not result in outright rejection of a manuscript, it may well influence the reviewer's and editor's overall impression of the manuscript. It has been shown that a well written manuscript has a better chance of being accepted.

4. Insufficient problem statement. It is important to clearly define and appropriately frame the study's question.

5. Methods not described in detail. Details are insufficient to repeat the results. The study design, apparatus used, and procedures followed must be made clear. In some cases it might be better to put too much information into the methods section rather than to put too little ; information deemed unnecessary can always be removed prior to publication.

6. Overinterpretation of results. Some reviewers have indicated that a clear and "honest" approach to the interpretation of the results is likely to increase the chances of a manuscript being accepted. Identify possible biases and confounding variables, both during the design phase of the study and the interpretation of the results. Describe experimental results concisely.

7. Inappropriate or incomplete statistics. Using inappropriate statistical methods and overstating the implications of the results is a common error. Use an appropriate test and do not make the statistics too complicated. Quantify and present findings with appropriate indicators of measurement error or uncertainty (such as confidence intervals).

8. Unsatisfactory or confusing presentation of data in tables or figures. The tables or figures do not conform in style and quantity to the journal's guidelines and are cluttered with numbers. Make tables and graphs easy to read. Some editors may start by looking quickly at the tables, graphs, and figures to determine if the manuscript is worth considering.

9. Conclusions not supported by data. Make sure your conclusions are not overstated, are supported, and answer the study's questions. Be sure to provide alternative explanations, and do not simply restate the results.

10. Incomplete, inaccurate, or outdated review of the literature. Be sure to conduct a complete literature search and only list references relevant to the study. The reviewers of your manuscript will be experts in the field and will be aware of all the pertinent research conducted.

11. Author unwilling to revise the manuscript to address reviewer's suggestions. This can easily be resolved. Taking the reviewers' suggestions into account when revising your manuscript will nearly always result in a better manuscript. If the editor indicates willingness to evaluate a revision, it means the manuscript may be publishable if the reviewers' concerns could be addressed satisfactorily.

full version - see 2008 JUN 